\title{
Mean platelet volume, red cell distribution width, neutrophil to lymphocyte ratio and platelet to lymphocyte ratio in the diagnosis of acute appendicitis
}

\author{
Osman Toktas $^{1^{*}}$ and Mehmet Aslan ${ }^{2}$ \\ ${ }^{1}$ Yuzuncu Yil University, Medical Faculty, Department of General Surgery, Van, Turkey \\ ${ }^{2} Y$ uzuncu Yil University, Medical Faculty, Department of Internal Medicine, Van, Turkey
}

\begin{abstract}
Mean platelet volume (MPV), red cell distribution width (RDW), platelet to lymphocyte ratio (PLR) and neutrophil to lymphocyte ratio (NLR) have been seperately reported to be laboratory markers in several inflammatory diseases, including acute appendicitis. However, the results of these studies are conflicting. The aim of this study was to simultaneously investigate if MPV, RDW, PLR and NLR have a diagnostic role in the diagnosis of acute appendicitis and also the relationship between these systemic inflammation marker and leukocyte count.

Thirty patients with acute appendicitis and 30 age-matched healthy subjects. RDW, MPV, neutrophils, lymphocytes and platelet counts were evaluated with complete blood count. The NLR and PLR were calculated as the ratios of the neutrophils and platelets to the lymphocytes.

NLR and leukocyte count were significantly higher in the acute appendicitis patients compared to controls (both $\mathrm{p}<0.001)$, while RDW levels were significantly lower $(p=0.041)$. There were no statistically significant differences regarding platelet numbers, MPV levels and PLR between acute appendicitis and healthy subjects (All; $\mathrm{p}>0.05$ ). There was a significant correlation between leukocyte count and NLR ( $<<0.01, r=0.365)$. However, leukocyte count was not correlated with RDW and MPV levels (all; $\mathrm{p}>0.05$ ).

The current study is the first to investigate MPV, RDW, PLR and NLR in acute appendicitis patients. We found significantly increased NLR and leukocyte count in acute appendicitis patients compared to healthy subjects. Combined use of NLR and RDW values along with leukocyte count and other clinical assessment could help the diagnostic process of acute appendicitis.
\end{abstract}

Key Words: Acute appendicitis, neutrophil to lymphocyte ratio, mean platelet volume, red cell distribution width, platelet number

\section{Introduction}

Acute appendicitis (AA) is one of the most common surgical disease of the abdomen, but the diagnosis of the disease in an emergency setting still presents some challenges (1). The diagnosis of AA is based on a brief history of abdominal pain, nausea, migration of pain to the right iliac fossa, and signs of local peritonitis; diagnostic certainty based on these symptoms ranges from $70 \%$ to $80 \%(1,2)$. The clinical diagnosis is often not easy even for veteran surgeons, as evidenced by the high rate of false explorations, which commonly reaches $20 \%$ to $30 \%$ (1). It leads to complications like perforation, abscess, sepsis and intestinal obstruction, if diagnosis of acute appendicitis is being late (3). Imaging modalities such as ultrasonography and computerized tomography, as well as diagnostic laparoscopy and recent laboratory tests have been increasingly used for quick and correct diagnosis (4). However, they are not enough (5).

Recently some studies have investigated various parameters like C-reactive protein, white blood cell count, lymphocyte/leukocyte rate, erythrocyte sedimentation rate, procalcitonin, fibrinogen, alpha 2- macroglobulin, alpha 1-antitrypsin and DLactate for the diagnosis of AA (2) however none of them were commonly accepted. So, simple, widely valid, inexpensive, and time-saving new laboratory methods that can be implemented anywhere are essentially for the diagnosis of AA.

Mean platelet volume (MPV) is a common test that is part of complete blood count (6). MPV is

*Corresponding Author: Asst. Prof. MD. Osman Toktas, Yuzuncu Yil University, Medical Faculty Department of General Surgery, 65000, Van, Turkey, Tel: +90 (432) 21504 73, Fax: +90 (432) 21675 19, E-mail: osmantoktas@windowslive.com 
reportedly associated with the platelet function. The length of the platelet is linked with the activity and the function of the platelet; bigger platelets are more active than small platelets (7). MPV has been shown to reflect inflammatory burden. Additionally, the relationship between MPV values and AA has been investigated in different clinical studies yet the results are inconsistent (8-11).

Red cell distribution width (RDW) is an easy-tomeasure part of complete blood count, showing variability of size of circulating erythrocytes. Enhanced RDW levels are related to disrupted erythropoiesis or erythrocyte degradation (12). Inflammation might disrupt red blood cell maturation, cell membrane damage throughly, causing RDW to increment. Different studies have reported that increased RDW levels, even within the normal reference range, were associated with unfavorable clinical outcomes in cases with cardiovascular diseases, type 2 diabetes mellitus, pulmonary thromboembolism and ischemic stroke $(13,14)$. Nowadays, a number of variables have been studied as an inflammatory marker in AA $(8,15,16)$ however the results are inconsistent.

Several authors have reported that an increased leukocyte count is used as diagnostic tool in AA. However, its sensitivity is low so leukocyte count is not diagnostic $(17,18)$. Nowadays, some authors demonstrated that the neutrophil to lymphocyte ratio (NLR) is a predictor of inflammation and helpful in the preoperative diagnosis of AA (1921).

To the best of our knowledge, MPV levels, RDW, NLR and PLR and in patients with AA have not simultaneouly been reported. The aim of this study was to simultaneously investigate if MPV, RDW and NLR have a diagnostic role in the diagnosis of $\mathrm{AA}$ and additionally the relationship between these systemic inflammation markers and leukocyte count.

\section{Materials and methods}

Subjects: The retrospective study was conducted at the Yuzuncu Yil University Medical Faculty Department of Surgery. Thirty cases (8 females and 22 males; mean age: $28 \pm 9$ years) with AA were enrolled in this study.

Controls consisted of 30 healthy subjects ( 9 females and 21 males; mean age: $29 \pm 10$ years) selected from a group of healthy subjects with no history of previous disease. All control groups were selected from healthy adults of similar age who applied to check-up clinic.

None of the cases had active infection, hypertension, diabetes mellitus, hyperlipidemia, chronic respiratory diseases, rheumatoid arthritis, cirrhosis, renal disease, coronary heart disease, cerebrovascular disease and malignant tumor.

The study protocol was carried out in accordance with the Helsinki Declaration as revised in 2000. All subjects were informed about the study protocol and written consent was obtained from each participant.

Mean platelet volume and platelet-tolymphocyte and neutrophil-to-lymphocyte ratios: Complete blood counts, including total white blood cells, neutrophils, lymphocytes, RDW, platelets and MPV values, were obtained using an automatic blood counter (BeckmanCoulter, LH 780, USA). The MPV was measured from a blood sample that was collected in a dipotassium EDTA tube. The NLR and PLR were calculated as the ratios of the neutrophils and platelets to the lymphocytes, and both were obtained from the automated blood sample that was acquired upon admission to the study.

Statistical analysis: The results were expressed as the meantstandard deviation. Continuous variables were compared using Student's test. Qualitative variables were assessed by Chi-square test. Correlation analyses were performed using Pearson's correlation test. The results were considered to be statistically significant when the $\mathrm{p}$ value was less than 0.05 . The data were analyzed using SPSS ${ }^{\circledR}$ for Windows (Version 20.0. Armonk, NY: IBM Corp.).

\section{Results}

The clinical and demographic data of the study population are shown in Table 1 . There was no statistically significant difference between two groups with regard to age and gender $(p>0.05)$ (Table 1).

The mean age of the AA cases was $28 \pm 9$ years, and the mean age of control subjects was $29 \pm 10$ years. Of the 30 acute appendicitis cases, 8 were female and 22 were male. Of the 30 control subjects, 9 were female and 21 were male (Table 1).

NLR and leukocyte count were significantly higher in the AA cases compared to controls (both $\mathrm{p}<0.01$ ), while RDW levels were significantly lower $(p=0.041)$. There was no statistically 
Table 1. Mean platelet volume, red cell distribution width, platelet number and neutrophil to lymphocyte ratio in acute appendicitis and control groups

\begin{tabular}{llll}
\hline Parameters & Acute appendicitis $(\mathrm{n}=30)$ & Controls $(\mathrm{n}=30)$ & $\mathrm{p}$ \\
\hline Age (Years) & $28 \pm 9$ & $29 \pm 10$ & 0.657 \\
Sex $($ Female/male) & $8 / 22$ & $9 / 21$ & 0.774 \\
Platelet number $\left(10^{3} / \mathrm{mm}^{3}\right)$ & $256 \pm 71$ & $286 \pm 79$ & 0.131 \\
Mean platelet volume (fL) & $8.08 \pm 0.84$ & $8.13 \pm 1.03$ & 0.846 \\
RDW (\%) & $13.07 \pm 0.96$ & $13.83 \pm 1.71$ & 0.041 \\
Neutrophil count $\left(\mathrm{mm}^{3}\right)$ & $1035 \pm 353$ & $379 \pm 104$ & 0.001 \\
Lymphocyte count $\left(\mathrm{mm}^{3}\right)$ & $228 \pm 100$ & $280 \pm 72$ & 0.026 \\
NLR (\%) & $6.11 \pm 4.76$ & $1.44 \pm 054$ & 0.001 \\
PLR (\%) & $1.40 \pm 0.96$ & $1.09 \pm 0.49$ & 0.130 \\
Leukocyte count $\left(\mathrm{mm}^{3}\right)$ & $14146 \pm 3326$ & $7650 \pm 1917$ & 0.001 \\
\hline
\end{tabular}

Values are mean \pm SD, RDW: Red cell distribution width, NLR: Neutrophil to lymphocyte ratio, PLR: Platelet to lymphocyte ratio

significant difference regarding platelet numbers, PLR and MPV values between AA and healthy subjects $(\mathrm{p}>0.05)($ Table 1$)$.

There was a significant correlation between leukocyte count and NLR $(\mathrm{p}<0.01, \mathrm{r}=0.365)$. However, leukocyte count was not correlated with RDW, PLR and MPV values ( $p>0.05)$.

\section{Discussion}

This study is the first to demonstrate MPV, RDW, PLR and NLR in AA cases. AA cases were significantly higher NLR and leukocyte count than healthy controls, while RDW levels were significantly lower and that there is important association between the leukocyte count and NLR in these cases.

The pathophysiology of AA is characterized by the mucosal ischemia of the appendix that results from ongoing mucus secretion from the appendiceal mucosa distal to an obstruction of the lumen, increase intraluminal pressure and, subsequent, venous flow is stopped. Luminal pressure exceeds $85 \mathrm{mmHg}$, venules that drain the appendix become thrombosed and, in the setting of continued arteriolar in flow, vascular congestion and engorgement of the appendix become manifest (2).

Different diagnostic methods are used for appendicitis. Severe morbidity and even death may occur when the diagnosis is delayed. Therefore, the first aim of surgeons must be right and earlier diagnosis. However, there is no laboratory value for right and exact diagnosis of appendicitis. In medical practice, leukocyte and neutrophil count are the most widely used laboratory parameters in the diagnosis of appendicitis, yet their specificity and sensitivity values are poor.

Leukocyte count is most constantly used to diagnose AA. Different reports have suggested that increased leukocyte count is generally the earliest laboratory measure to indicate inflammation of the appendix, and plenty cases with AA present with leukocytosis $(1,11)$. A relationship among the increase of leukocyte count and appendicitis diagnosis have been shown. It has been reported that in cases with AA, the sensitivity of leukocyte is between $60-87 \%$, and specificity $53-100 \%$ (22). In agreement with the literature, we observed that leukocyte count was significantly higher in AA compared to healthy subjects.

Neutrophil is generally associated with bacterial infections. There are very little studies on this subject, yet it is reported that NLR appears to have greater diagnostic accuracy than traditional diagnostic laboratory tests (either white blood cell or C-reactive protein alone). Additionally, it is reported that NLR on admission to the hospital is an independent predictor of positive appendicitis histology (19). Nowadays, some authors report that the NLR is a predictor of inflammation and helpful in the preoperative diagnosis of AA $(21,23)$. In the study of Yazici et al. (21) it was reported that the sensitivity is greatest when NLR is $>3.5$. Goodman et al. (23) advocated the use of NLR as a diagnostic tool in adults, and a ratio above 3.5 was stated as optimally diagnostic for appendicitis. Significant rise in NLR over 3 in cases with appendicitis may be explained by neutrophilia together with lymphopenia in cases with gangrenous type appendicitis (24). Zahorec 
(25) suggested the use of the NLR as a fast and easy parameter for systemic inflammation and stress in critically ill cases. In our study, we observed significant increase in NLR in acute appendicitis cases than healthy controls.

MPV is a parameter calculated and provided by automatic blood count equipment during routine blood counts. Platelet activation is a link in the pathophysiology of diseases prone to thrombosis and inflammation. Some studies have assessed the role of the MPV values in cases with AA (8-11) however these studies are contradictory. While some authors reported lowered MPV values in patients with acute appendicitis (8), other studies have reported increased MPV values (9). But, others have reported no significance in MPV values $(10,11)$. In the present study, we did not find an important difference in the MPV values among AA and healthy subjects.

As a combination of both platelet and lymphocyte counts, the PLR was introduced as a potential marker to establish inflammation in cardiac and oncologic disorders (26). However, any studies till date have been investigated in AA. In the present study, we did not find a important difference in the PLR values in AA compared than healthy subjects. This is the first report to evaluate the PLR in AA.

RDW is an automated measure of the variability of red blood cell size (27). RDW is a widely used laboratory parameter for the quantification of the extent of erythrocyte anisocytosis. Some studies have shown that RDW levels are an independent variant of prognosis in cases with cardiovascular diseases, type 2 diabetes mellitus, pulmonary thromboembolism and ischemic stroke $(13,14)$. Additionally, It was found to be related to mortality and other severe adverse outcomes in infectious diseases (28). Nowadays, different authors have been studied as an inflammatory marker in $\mathrm{AA}(8,15,16)$ however the results are limited and contradictory. Narci et al. (15) observed that RDW levels were significantly lower in cases with AA than healthy subjects. In contrast, Bozlu et al. (16) found the higher levels of RDW in children with AA compared with control groups. On the other hand, Tanrikulu et al. (8) did not find an important difference in the RDW values among AA and healthy subjects. In our study, RDW levels were significantly lower in the AA patients than healthy controls.

The present study is the first to investigate MPV, RDW, PLR and NLR in AA cases. We found significantly increased NLR and leukocyte count in AA cases compared to healthy subjects while
RDW levels were significantly lower. Combined use of NLR and RDW values along with leukocyte count and other clinical assessment could help the diagnostic process of AA. Further studies are necessary to confirm these findings.

Conflict of interest statement: The authors declare that there are no conflicts of interest.

Financial Disclosure: The authors declared that this study has received no financial support.

Acknowledgements: O.T and M.A: contributed equally to this manuscript

\section{References}

1. Andersson RE. Meta-analysis of the clinical and laboratory diagnosis of appendicitis. Br J Surg 2004; 91: 28-37.

2. Karagulle E, Turk E, Ezer A, et al. Value of Plasma Viscosity in Acute Appendicitis: a Preliminary Study. J Med Med Sci 2010; 1: 423425.

3. Graff L, Russell J, Seashore J, et al. Falsenegative and false-positive errors in abdominal pain evaluation: failure to diagnose acute appendicitis and unnecessary surgery. Acad Emerg Med 2000; 7: 1244-1255.

4. Orr RK, Porter D, Hartman D. Ultrasonography to evaluate adults for appendicitis: decision making based on meta-analysis and probabilistic reasoning. Acad Emerg Med 1995; 2: 644-650.

5. Lessin MS, Chan M, Catallozzi M, et al. Selective use of ultrasonography for acute appendicitisin children. Am J Surg 1999; 177: 193-196.

6. Park Y, Schoene N, Harris W. Mean platelet volume as an indicator of platelet activation: methodological issues. Platelets 2002; 13: 301306.

7. Thompson CB, Jakubowski JA, Quinn PG, Deykin D, Valeri CR. Platelet size as a determinant of platelet function. J Lab Clin Med 1983; 101: 205-213.

8. Tanrikulu CS, Tanrikulu Y, Sabuncuoglu MZ, et al. Mean platelet volume and red cell distribution width as a diagnostic marker in acute appendicitis. Iran Red Crescent Med J 2014; 16: e10211.

9. Uyanik B, Kavalci C, Arslan ED, et al. Role of mean platelet volume in diagnosis of childhood acute appendicitis. Emerg Med Int 2012; 2012: 823095.

10. Bilici S, Sekmenli T, Göksu M, Melek M, Avci V. Mean platelet volume in diagnosis of acute appendicitis in children. Afr Health Sci 2011; 11: 427-432.

11. Erdem H, Aktimur R, Cetinkunar $S$, et al. Evaluation of mean platelet volume as a

East J Med Volume:22, Number:1, January-March/2017 
diagnostic biomarker in acute appendicitis. Int $\mathrm{J}$ Clin Exp Med 2015; 8: 1291-1295.

12. Oztürk ZA, Unal A, Yigiter R, et al. Is increased red cell distribution width (RDW) indicating the inflammation in Alzheimer's disease (AD)? Arch Gerontol Geriatr 2013; 56: 50-54.

13. Felker GM, Allen LA, Pocock SJ, et al. Red cell distribution width as a novel prognostic marker in heart failure: data from the CHARM Program and the Duke Databank. J Am Coll Cardiol 2007; 50: 40-47.

14. Malandrino N, Wu WC, Taveira TH, et al. Association between red blood cell distribution width and macrovascular and microvascular complications in diabetes. Diabetologia 2012; 55: 226-235.

15. Narci H, Turk E, Karagulle E, Togan T, Karabulut K. The role of red cell distribution width in the diagnosis of acute appendicitis: a retrospective case-controlled study. World J Emerg Surg 2013; 8: 46-50.

16. Bozlu G, Taskinlar H, Unal S, et al. Diagnostic value of red blood cell distribution width in pediatric acute appendicitis. Pediatr Int 2016; 58: 202-205.

17. Yang HR, Wang YC, Chung PK, et al. Role of leukocyte count, neutrophil percentage, and Creactive protein in the diagnosis of acute appendicitis in the elderly. Am Surg 2005; 71: 344-347.

18. Hallan S, Asberg A, Edna TH. Additional value of biochemical tests in suspected acute appendicitis. Eur J Surg 1997; 163: 533-538.

19. Shimizu T, Ishizuka M, Kubota K. A lower neutrophil to lymphocyte ratio is closely associated with catarrhal appendicitis versus severe appendicitis. Surg Today 2016; 46: 84-89.
20. Markar SR, Karthikesalingam A, Falzon A, Kan $Y$. The diagnostic value of neutrophil: lymphocyte ratio in adults with suspected acute appendicitis. Acta Chir Belg 2010; 110: 543-547.

21. Yazici M, Ozkisacik S, Oztan MO, Gürsoy H. Neutrophil/lymphocyte ratio in the diagnosis of childhood appendicitis. Turk J Pediatr 2010; 52: 400-403.

22. Acikgoz N, Karincaoglu Y, Ermis N, et al. Increased mean platelet volume in Behçet's disease with thrombotic tendency. Tohoku J Exp Med 2010; 221: 119-123.

23. Goodman DA, Goodman CB, Monk JS. Use of the neutrophil:lymphocyte ratio in the diagnosis of appendicitis. Am Surg 1995; 61: 257-259.

24. Jahangiri M, Wyllie JH. Peripheral blood lymphopenia in gangrenous appendicitis. BMJ 1990; 301: 215.

25. Zahorec R. Ratio of neutrophil to lymphocyte counts rapid and simple parameter of systemic inflammation and stress in critically ill. Bratisl Lek Listy 2001; 102: 5-14.

26. Ugur M, Gul M, Bozbay M, et al. The relationship between platelet to lymphocyte ratio and the clinical outcomes in ST elevation myocardial infarction underwent primary coronary intervention. Blood Coagul Fibrinolysis 2014; 6: 212-216.

27. Lou Y, Wang M, Mao W. Clinical usefulness of measuring red blood cell distribution width in patients with Hepatitis B. PLoS One 2012; 7: e37644.

28. Chen B, Ye B, Zhang J, Ying L, Chen Y. RDW to Platelet Ratio: A novel noninvasive index for predicting hepatic fibrosis and cirrhosis in chronic hepatitis. B. PLoS One 2013; 8: e68780. 